Published in: Frontiers of Mathematics in China 1 (2006), 1: 73-97.

\title{
Branching processes with immigration and related topics
}

\author{
Zenghu $\mathrm{LI}^{1}$ \\ School of Mathematical Sciences, Beijing Normal University, Beijing 100875, China
}

\begin{abstract}
This is a survey on recent progresses in the study of branching processes with immigration, generalized Ornstein-Uhlenbeck processes and affine Markov processes. We mainly focus on the applications of skew convolution semigroups and the connections in those processes.
\end{abstract}

Keywords: Branching process, immigration, measure-valued process, affine process, Ornstein-Uhlenbeck process, skew convolution semigroup, stochastic equation, fluctuation limit.

Mathematics Subject Classification (2000): 60J80, 60F05, 60H20, 60K37

\section{Introduction}

Let $\mathbb{N}:=\{0,1,2, \cdots\}$ and let $\{\xi(n, i): n, i=1,2, \cdots\}$ be a sequence of $\mathbb{N}$-valued i.i.d. random variables. Let $x(0)$ be an $\mathbb{N}$-valued random variable which is independent of $\{\xi(n, i)\}$. A GaltonWatson branching process (GW-process) $\{x(n): n=0,1,2, \cdots\}$ is defined inductively by

$$
x(n)=\sum_{i=1}^{x(n-1)} \xi(n, i), \quad n=1,2, \cdots .
$$

This process is a mathematical representation of the random evolution of an isolated population. We refer the reader to Athreya and Ney [1] and Harris [29] for the theory of branching processes.

A useful and realistic modification of the above scheme is the addition of the possibility of immigration into the population. From the point of applications, the immigration processes are clearly of great importance. Let $\{\eta(n): n=1,2, \cdots\}$ be another sequence of $\mathbb{N}$-valued i.i.d. random variables which are independent of $\{\xi(n, i)\}$. Let $y(0)$ be an $\mathbb{N}$-valued random variable independent of $\{\xi(n, i)\}$ and $\{\eta(n)\}$. We can define a Galton-Watson branching process with immigration (GWI-process) $\{y(n): n=0,1,2, \cdots\}$ by

$$
y(n)=\sum_{i=1}^{y(n-1)} \xi(n, i)+\eta(n), \quad n=1,2, \cdots ;
$$

see, e.g., 11, p.263]. The intuitive meaning of the process is clear from the construction (1.2). Let $g(\cdot)$ and $h(\cdot)$ be the generating function of $\{\xi(n, i)\}$ and $\{\eta(n)\}$, respectively. Because of

\footnotetext{
${ }^{1}$ E-mail: lizh@bnu.edu.cn
} 
the independence of the random variables $\{\xi(n, i), \eta(n): n, i=1,2, \cdots\}$ it is easy to see that $\{y(n)\}$ is a discrete-time Markov chain with one-step transition matrix $P(i, j)$ defined by

$$
\sum_{j=0}^{\infty} P(i, j) z^{j}=g(z)^{i} h(z), \quad 0 \leq z \leq 1 ; i=0,1,2, \cdots
$$

The purpose of this survey is to give a brief introduction to the recent progresses in the study of branching processes with immigration and related topics. We shall be concerned with continuous state branching processes (CB processes), CB processes with immigration (CBI processes), measure-valued branching processes (MB processes), Dawson-Watanabe superprocesses, immigration superprocesses, generalized Ornstein-Uhlenbeck processes, and affine processes. The basic mathematical structures of those processes already exist in (1.1)-(1.3). Our emphasis is on the applications of skew convolution semigroups and the connections in those processes. This is an ongoing research topic of the Probability Group in Beijing Normal University. Other topics where our Group has been involved include interacting particle systems, ergodic and spectral theory, probabilistic and functional inequalities, large and moderate deviations and so on. We refer the reader to Chen [6, 7, 8] and Wang [67, 68, 69] for some recent results of the Group on those topics.

Let us introduce some notation which will be used throughout the survey. Given a metrizable topological space $E$, we denote by $\mathscr{B}(E)$ its Borel $\sigma$-algebra. Let $B(E)$ the space of bounded real $\mathscr{B}(E)$-measurable functions on $E$ and $C(E)$ the subset of $B(E)$ of continuous functions. Let $M(E)$ be the space of finite Borel measures on $E$ endowed with the topology of weak convergence. For $f \in B(E)$ and $\mu \in M(E)$, let $\mu(f)=\int_{E} f d \mu$. Let $\delta_{x}$ denote the unit mass concentrated at $x \in E$. For any integer $m \geq 1$ let $C^{m}\left(\mathbb{R}^{d}\right)$ denote the set of smooth functions on the Euclidean space $\mathbb{R}^{d}$ with all partial derivatives up to the $m$ th order belonging to $C\left(\mathbb{R}^{d}\right)$. Let $C^{\infty}\left(\mathbb{R}^{d}\right)=\bigcap_{m=1}^{\infty} C^{m}\left(\mathbb{R}^{d}\right)$.

\section{Skew convolution semigroups and examples}

Let $(S,+)$ be a metrizable abelian semigroup, that is, $S$ is a metrizable topological space and there is a composition law $+: S^{2} \rightarrow S$ which is associative, commutative and continuous. For two Borel probability measures $\mu$ and $\nu$ on $S$, the image of the product measure $\mu \times \nu$ under the composition law is called the convolution of $\mu$ and $\nu$ and is denoted by $\mu * \nu$. Suppose that $\left(Q_{t}\right)_{t \geq 0}$ is a Borel Markov transition semigroup on $S$ satisfying $Q_{t}(0, \cdot)=\delta_{0}$ and the branching property

$$
Q_{t}\left(x_{1}+x_{2}, \cdot\right)=Q_{t}\left(x_{1}, \cdot\right) * Q_{t}\left(x_{2}, \cdot\right), \quad t \geq 0, x_{1}, x_{2} \in S .
$$

Given $t \geq 0$ and a Borel measure $\mu$ on $S$ we define the measure $\mu Q_{t}$ by

$$
\mu Q_{t}(A)=\int_{S} Q_{t}(x, A) \mu(d x), \quad A \in \mathscr{B}(S) .
$$

Lemma 2.1 For any Borel probability measures $\mu$ and $\nu$ on $S$ we have

$$
(\mu * \nu) Q_{t}=\left(\mu Q_{t}\right) *\left(\nu Q_{t}\right), \quad t \geq 0 .
$$


Proof. Let $f \in B(S)$. From the branching property it follows that

$$
\begin{aligned}
\int_{S} Q_{t} f(x)(\mu * \nu)(d x) & =\int_{S} \mu(d x) \int_{S} Q_{t} f(x+y) \nu(d y) \\
& =\int_{S} \mu(d x) \int_{S} \nu(d y) \int_{S} f(z) Q_{t}(x+y, d z) \\
& =\int_{S} \mu(d x) \int_{S} \nu(d y) \int_{S} Q_{t}\left(x, d z_{1}\right) \int_{S} f\left(z_{1}+z_{2}\right) Q_{t}\left(y, d z_{2}\right) \\
& =\int_{S}\left(\mu Q_{t}\right)\left(d z_{1}\right) \int_{S} f\left(z_{1}+z_{2}\right)\left(\nu Q_{t}\right)\left(d z_{2}\right) \\
& =\int_{S} f(z)\left[\left(\mu Q_{t}\right) *\left(\nu Q_{t}\right)\right](d z)
\end{aligned}
$$

Then we have the equality (2.2).

Theorem 2.1 Suppose that $\left(\gamma_{t}\right)_{t \geq 0}$ is a family of Borel probability measures on $S$. Then

$$
Q_{t}^{\gamma}(x, \cdot):=Q_{t}(x, \cdot) * \gamma_{t}(\cdot), \quad x \in S, t \geq 0
$$

defines a Borel kernel on $S$ and $\left(Q_{t}^{\gamma}\right)_{t \geq 0}$ form a transition semigroup if and only if

$$
\gamma_{r+t}=\left(\gamma_{r} Q_{t}\right) * \gamma_{t}, \quad r, t \geq 0
$$

Proof. It is easy to show that $Q_{t}^{\gamma}(x, d y)$ is a Borel kernel on $S$. Then we only need to prove that (2.4) is equivalent to the Chapman-Kolmogorov equation

$$
\int_{S} f(y) Q_{r+t}^{\gamma}(x, d y)=\int_{S} Q_{r}^{\gamma}(x, d y) \int_{S} f(z) Q_{t}^{\gamma}(y, d z), \quad f \in B(S) .
$$

If (2.5) holds, we may apply this equation with $x=0$ to see that

$$
\begin{aligned}
\int_{S} f(z) \gamma_{r+t}(d z) & =\int_{S} \gamma_{r}(d y) \int_{S} f(z) Q_{t}^{\gamma}(y, d z) \\
& =\int_{S} \gamma_{r}(d y) \int_{S} Q_{t}\left(y, d z_{1}\right) \int_{S} f\left(z_{1}+z_{2}\right) \gamma_{t}\left(d z_{2}\right) \\
& =\int_{S}\left(\gamma_{r} Q_{t}\right)\left(d z_{1}\right) \int_{S} f\left(z_{1}+z_{2}\right) \gamma_{t}\left(d z_{2}\right) .
\end{aligned}
$$

Then (2.4) holds. Conversely, if (2.4) holds, we have

$$
\begin{aligned}
\int_{S} f(z) Q_{r+t}^{\gamma}(x, d z) & =\int_{S} Q_{r+t}\left(x, d z_{1}\right) \int_{S} f\left(z_{1}+z_{2}\right) \gamma_{r+t}\left(d z_{2}\right) \\
& =\int_{S} Q_{r}(x, d y) \int_{S} Q_{t}\left(y, d z_{1}\right) \int_{S}\left(\gamma_{r} Q_{t}\right)\left(d z_{2}\right) \int_{S} f\left(z_{1}+z_{2}+z_{3}\right) \gamma_{t}\left(d z_{3}\right) \\
& =\int_{S} Q_{r}^{\gamma}(x, d y) \int_{S} Q_{t}\left(y, d z_{2}\right) \int_{S} f\left(z_{2}+z_{3}\right) \gamma_{t}\left(d z_{3}\right) \\
& =\int_{S} Q_{r}^{\gamma}(x, d y) \int_{S} f(z) Q_{t}^{\gamma}(y, d z)
\end{aligned}
$$


That proves the Chapman-Kolmogorov equation (2.5).

We call $\left(\gamma_{t}\right)_{t \geq 0}$ a skew convolution semigroup (SC-semigroup) associated with $\left(Q_{t}\right)_{t \geq 0}$ if it satisfies (2.4); see $\mathrm{Li}$ [44, 50]. The the kernels $Q_{t}^{\gamma}(x, d y)$ defined by (2.3) give an abstract formulation of the expression (1.3). In particular, if $Q_{t}$ is the identity operator for every $t \geq 0$, the SC-semigroup defined by (2.4) becomes a standard convolution semigroup and $\left(Q_{t}^{\gamma}\right)_{t \geq 0}$ is the transition semigroup of a Lévy process. We refer the reader to Bertoin [3] and Sato 61] for the theory of Lévy processes. The general formulae (2.3) and (2.4) include many additional mathematical contents, which are illustrated by the following examples.

Example 2.1 In the particular case $S=\mathbb{R}_{+}$, a Markov process with transition semigroup $\left(Q_{t}\right)_{t \geq 0}$ is called a $C B$-process and a Markov process with transition semigroup $\left(Q_{t}^{\gamma}\right)_{t \geq 0}$ is called a $C B I$-process; see [39, 65].

Example 2.2 If $S=M(E)$ is the space of all finite Borel measures on a metrizable space $E$, the semigroup $\left(Q_{t}\right)_{t \geq 0}$ corresponds to an MB-process, of which the Dawson-Watanabe superprocess is a special case; see 9]. A Markov process with state space $M(E)$ is naturally called an immigration superprocess associated with $\left(Q_{t}\right)_{t \geq 0}$ if it has transition semigroup $\left(Q_{t}^{\gamma}\right)_{t \geq 0}$; see 44, 45, 50].

Example 2.3 Let us consider the case where $S=H$ is a real separable Hilbert space and $Q_{t}(x, \cdot) \equiv \delta_{T_{t} x}$ for a strongly continuous semigroup of bounded linear operators $\left(T_{t}\right)_{t \geq 0}$ on $H$. In this case, $\left(Q_{t}^{\gamma}\right)_{t \geq 0}$ is called a generalized Mehler semigroup associated with $\left(T_{t}\right)_{t \geq 0}$, which corresponds to a generalized Ornstein-Uhlenbeck process (OU-process). This formulation of the processes was given by Bogachev et al. 44; see also [16, 25].

Example 2.4 If $S=\mathbb{R}_{+}^{m} \times \mathbb{R}^{n}$ for integers $m \geq 0$ and $n \geq 0$, the transition semigroup $\left(Q_{t}^{\gamma}\right)_{t \geq 0}$ corresponds to an affine process. The affine Markov processes were introduced in mathematical finance; see, e.g., [15, 19].

\section{Continuous state branching processes with immigration}

There is a rich literature in the study of CB- and CBI-processes. In particular, the class of CBIprocesses was characterized completely by Kawazu and Watanabe [39]. Let $F$ be a function defined by

$$
F(\lambda)=b \lambda+\int_{0}^{\infty}\left(1-e^{-\lambda u}\right) m(d u), \quad \lambda \geq 0,
$$

where $b \geq 0$ is a constant and $u m(d u)$ is a finite measure on $(0, \infty)$. Let $R$ be given by

$$
R(\lambda)=\beta \lambda-\alpha \lambda^{2}-\int_{0}^{\infty}\left(e^{-\lambda u}-1+\lambda u\right) \mu(d u), \quad \lambda \geq 0
$$

where $\beta \in \mathbb{R}$ and $\alpha \geq 0$ are constants and $\left(u \wedge u^{2}\right) \mu(d u)$ is a finite measure on $(0, \infty)$. We can define a transition semigroup $\left(P_{t}\right)_{t \geq 0}$ on $\mathbb{R}_{+}$by

$$
\int_{0}^{\infty} e^{-\lambda y} P_{t}(x, d y)=\exp \left\{-x \psi_{t}(\lambda)-\int_{0}^{t} F\left(\psi_{s}(\lambda)\right) d s\right\}, \quad \lambda \geq 0,
$$


where $\psi_{t}(\lambda)$ is the unique solution of

$$
\frac{d \psi_{t}}{d t}(\lambda)=R\left(\psi_{t}(\lambda)\right), \quad \psi_{0}(\lambda)=\lambda .
$$

A Markov process $\{y(t): t \geq 0\}$ with transition semigroup $\left(P_{t}\right)_{t \geq 0}$ is a special case of the CBIprocess defined in 39. Here (3.3) is the continuous time version of (1.3). Various limit theorems for the CBI-process have been established; see, e.g., [28, 48, 57, 58, and the references therein.

The connections between the GWI-processes and the CBI-processes were investigated in Kawazu and Watanabe [39. They showed that a CBI-processes arises as the high density limit in finite-dimensional distributions of a sequence of GWI-processes. Some simple conditions were given in $\mathrm{Li}$ [51] which ensure that the convergence of GWI-processes mentioned above holds on the space of càdlàg paths. Let $\left\{y_{k}(n): n \geq 0\right\}$ be a sequence of GWI-processes with parameters $\left\{\left(g_{k}, h_{k}\right)\right\}$ and $\left\{\gamma_{k}\right\}$ a sequence of positive numbers. For $0 \leq \lambda \leq k$ set

$$
F_{k}(\lambda)=\gamma_{k}\left[1-h_{k}(1-\lambda / k)\right]
$$

and

$$
R_{k}(\lambda)=k \gamma_{k}\left[(1-\lambda / k)-g_{k}(1-\lambda / k)\right]
$$

Let us consider the following conditions:

(3.A) As $k \rightarrow \infty$, we have $\gamma_{k} \rightarrow \infty$ and $\gamma_{k} / k \rightarrow$ some $\gamma_{0} \geq 0$.

(3.B) The sequence $\left\{F_{k}\right\}$ defined by (3.5) is uniformly Lipschitz on each bounded interval and converges as $k \rightarrow \infty$.

(3.C) The sequence $\left\{R_{k}\right\}$ defined by (3.6) is uniformly Lipschitz on each bounded interval and converges as $k \rightarrow \infty$.

Theorem 3.1 (51]) Suppose that conditions (3.A), (3.B) and (3.C) are satisfied. If $y_{k}(0) / k$ converges in distribution to $y(0)$, then $\left\{y_{k}\left(\left[\gamma_{k} t\right]\right) / k: t \geq 0\right\}$ converges in distribution on $D\left([0, \infty), \mathbb{R}_{+}\right)$to the CBI-process $\{y(t): t \geq 0\}$ corresponding to $(R, F)$.

Proof. We here only give a sketch and refer the reader to [51] for the details. Let $A$ denote the generator of the CBI-process. For $\lambda>0$ and $x \geq 0$ set $e_{\lambda}(x)=e^{-\lambda x}$ and let $D$ be the linear hull of $\left\{e_{\lambda}: \lambda>0\right\}$. For $\lambda>0$ we have

$$
A e_{\lambda}(x)=-e^{-\lambda x}[x R(\lambda)+F(\lambda)], \quad x \in \mathbb{R}_{+},
$$

and this equality determines the actions of $A$ on $D$ by linearity. Then we deduce that $D$ is a core of $A$. Note that $\left\{y_{k}(n) / k: n \geq 0\right\}$ is a Markov chain with state space $E_{k}:=\{0,1 / k, 2 / k, \cdots\}$ and one-step transition probability $Q_{k}(x, d y)$ determined by

$$
\int_{E_{k}} e^{-\lambda y} Q_{k}(x, d y)=g_{k}\left(e^{-\lambda / k}\right)^{k x} h_{k}\left(e^{-\lambda / k}\right)
$$

Then one checks that the (discrete) generator $A_{k}$ of $\left\{y_{k}\left(\left[\gamma_{k} t\right]\right) / k: t \geq 0\right\}$ is given by

$$
\begin{aligned}
A_{k} e_{\lambda}(x) & =\gamma_{k}\left[g_{k}\left(e^{-\lambda / k}\right)^{k x} h_{k}\left(e^{-\lambda / k}\right)-e^{-\lambda x}\right] \\
& =\gamma_{k}\left[\exp \left\{x k \alpha_{k}(\lambda)\left(g_{k}\left(e^{-\lambda / k}\right)-1\right)\right\} \exp \left\{\beta_{k}(\lambda)\left(h_{k}\left(e^{-\lambda / k}\right)-1\right)\right\}-e^{-\lambda x}\right],
\end{aligned}
$$


where

$$
\alpha_{k}(\lambda)=\left(g_{k}\left(e^{-\lambda / k}\right)-1\right)^{-1} \log g_{k}\left(e^{-\lambda / k}\right)
$$

and $\beta_{k}(\lambda)$ is defined by the same formula with $g_{k}$ replaced by $h_{k}$. Then we use the assumptions to show that

$$
A_{k} e_{\lambda}(x)=-e^{-\lambda x}\left[x \alpha_{k}(\lambda) S_{k}(\lambda)+x \gamma_{k}\left(\alpha_{k}(\lambda)-1\right) \lambda+H_{k}(\lambda)\right]+o(1),
$$

where

$$
H_{k}(\lambda)=\gamma_{k} \beta_{k}(\lambda)\left(1-h_{k}\left(e^{-\lambda / k}\right)\right)
$$

By elementary calculations we find that

$$
\alpha_{k}(\lambda)=1+\frac{1}{2}\left(1-g_{k}\left(e^{-\lambda / k}\right)\right)+o\left(1-g_{k}\left(e^{-\lambda / k}\right)\right),
$$

and so $\lim _{k \rightarrow \infty} \gamma_{k}\left(\alpha_{k}(\lambda)-1\right)=\gamma_{0} \lambda / 2$. It follows that

$$
\lim _{k \rightarrow \infty}\left[\alpha_{k}(\lambda) S_{k}(\lambda)+\gamma_{k}\left(\alpha_{k}(\lambda)-1\right) \lambda\right]=R(\lambda) .
$$

Then one shows that $\lim _{k \rightarrow \infty} H_{k}(\lambda)=\lim _{k \rightarrow \infty} F_{k}(\lambda)=F(\lambda)$. In view of (3.7) and (3.8) we get

$$
\lim _{k \rightarrow \infty} \sup _{x \in E_{k}}\left|A_{k} e_{\lambda}(x)-A e_{\lambda}(x)\right|=0
$$

for each $\lambda>0$. This clearly implies that

$$
\lim _{k \rightarrow \infty} \sup _{x \in E_{k}}\left|A_{k} f(x)-A f(x)\right|=0
$$

for each $f \in D$. That proves the desired convergence.

By the results of Li [42] it is easy to show that the limit functions of $\left\{F_{k}\right\}$ and $\left\{R_{k}\right\}$ always have representations (3.5) and (3.6), respectively. On the other hand, for any $(F, R)$ given by (3.1) and (3.2), there are sequences $\left\{\gamma_{k}\right\}$ and $\left\{\left(g_{k}, h_{k}\right)\right\}$ as above such that (3.A), (3.B) and (3.C) hold with $F_{k} \rightarrow F$ and $R_{k} \rightarrow R$; see [43, 51. Those results show the range of applications of Theorem 3.1 As consequences of the above theorem, Li [51] gave some generalizations of the Ray-Knight Theorems on Brownian local times; see also Le Gall and Le Jan [41. We remark that conditions (3.A), (3.B) and (3.C) parallel the sufficient conditions for the convergence of continuous-time and discrete state branching processes with immigration, see, e.g., 43]. In most cases, those conditions are easier to check than the sufficient conditions given by Kawazu and Watanabe [39], which involve complicated composition and convolution operations.

From (3.3) it is easy to see that the transition semigroup $\left(P_{t}\right)_{t \geq 0}$ is Fellerian, so the CBIprocess has a Hunt realization. A construction of the process was given in Dawson and Li [15] as the strong solution of a stochastic integral equation. Suppose that $(\Omega, \mathscr{F}, \mathscr{F} t, \mathbf{P})$ is a filtered probability space satisfying the usual hypotheses on which the following adapted objects are defined:

- a standard Brownian motion $\{B(t)\}$; 
- a Poisson random measure $N_{0}(d s, d \xi)$ on $\mathbb{R}_{+}^{2}$ with intensity $d s m(d \xi)$;

- a Poisson random measure $N_{1}(d s, d u, d \xi)$ on $\mathbb{R}_{+}^{3}$ with intensity $d s d u \mu(d \xi)$;

We assume that $\{B(t)\},\left\{N_{0}(d s, d \xi)\right\}$ and $\left\{N_{1}(d s, d u, d \xi)\right\}$ are independent of each other. Let $x(0)$ be a non-negative $\mathscr{F}_{0}$-measurable random variable satisfying $\mathbf{E}[x(0)]<\infty$. We consider the stochastic integral equation

$$
\begin{aligned}
x(t)= & x(0)+\int_{0}^{t}(b+\beta x(s)) d s+\int_{0}^{t} \sqrt{2 \alpha x(s)} d B(s) \\
& +\int_{0}^{t} \int_{0}^{\infty} \xi N_{0}(d s, d \xi)+\int_{0}^{t} \int_{0}^{x(s-)} \int_{0}^{\infty} \xi \tilde{N}_{1}(d s, d u, d \xi),
\end{aligned}
$$

where $\tilde{N}_{1}(d s, d u, d \xi)=N_{1}(d s, d u, d \xi)-d s d u \mu(d \xi)$.

Theorem 3.2 ([15]) There is a unique non-negative càdlàg process $\{x(t): t \geq 0\}$ such that equation (3.9) is satisfied a.s. for every $t \geq 0$.

The above theorem implies that (3.9) has a unique strong solution $\{x(t): t \geq 0\}$ and the solution is a strong Markov process. For $f \in C^{2}\left(\mathbb{R}_{+}\right)$we see from (3.9) and Itô's formula (see, e.g., [18, p.334-335]) that

$$
\begin{aligned}
f(x(t))= & f(x(0))+\int_{0}^{t} f^{\prime}(x(s))(b+\beta x(s)) d s+\text { martingale } \\
& +\int_{0}^{t} \int_{0}^{\infty} f^{\prime}(x(s)) \xi N_{0}(d s, d \xi)+\alpha \int_{0}^{t} f^{\prime \prime}(x(s)) x(s) d s \\
& +\int_{0}^{t} \int_{0}^{\infty}\left[f(x(s)+\xi)-f(x(s))-f^{\prime}(x(s)) \xi\right] N_{0}(d s, d \xi) \\
& +\int_{0}^{t} \int_{0}^{x(s-)} \int_{0}^{\infty}\left[f(x(s)+\xi)-f(x(s))-f^{\prime}(x(s)) \xi\right] N_{1}(d s, d u, d \xi) \\
= & f(x(0))+\int_{0}^{t} f^{\prime}(x(s))(b+\beta x(s)) d s+\text { martingale } \\
& +\alpha \int_{0}^{t} f^{\prime \prime}(x(s)) x(s) d s+\int_{0}^{t} d s \int_{0}^{\infty}[f(x(s)+\xi)-f(x(s))] m(d \xi) \\
& +\int_{0}^{t} d s \int_{0}^{\infty}\left[f(x(s)+\xi)-f(x(s))-f^{\prime}(x(s)) \xi\right] x(s) \mu(d \xi) .
\end{aligned}
$$

Then $\{x(t): t \geq 0\}$ has generator $A$ defined by

$$
\begin{aligned}
A f(x)= & \alpha x f^{\prime \prime}(x)+(b+\beta x) f^{\prime}(x)+\int_{0}^{\infty}[f(x+\xi)-f(x)] m(d \xi) \\
& +\int_{0}^{\infty}\left[f(x+\xi)-f(x)-f^{\prime}(x) \xi\right] x \mu(d \xi),
\end{aligned}
$$

so it is a CBI-process; see 39.

The approach of stochastic equations was also used in [15] to construct a general type of CBI-processes in random catalysts. Suppose we have the parameters $\left(a,\left(\alpha_{i j}\right),\left(b_{1}, b_{2}\right),\left(\beta_{i j}\right), m, \mu\right)$ such that 
- $a \in \mathbb{R}_{+}$is a constant;

- $\left(\alpha_{i j}\right)$ is a symmetric non-negative definite $(2 \times 2)$-matrix;

- $\left(b_{1}, b_{2}\right) \in \mathbb{R}_{+}^{2}$ is a vector;

- $\left(\beta_{i j}\right)$ is a $(2 \times 2)$-matrix with $\beta_{12}=0$;

- $m(d \xi)$ is a $\sigma$-finite measure on $\mathbb{R}_{+}^{2}$ supported by $\mathbb{R}_{+}^{2} \backslash\{0\}$ such that

$$
\int_{\mathbb{R}_{+}^{2}}\left[\xi_{1}+\xi_{2}\right] m(d \xi)<\infty
$$

- $\mu(d \xi)$ is a $\sigma$-finite measure on $\mathbb{R}_{+}^{2}$ supported by $\mathbb{R}_{+}^{2} \backslash\{0\}$ such that

$$
\int_{\mathbb{R}_{+}^{2}}\left[\left(\xi_{1} \wedge \xi_{1}^{2}\right)+\left(\xi_{2} \wedge \xi_{2}^{2}\right)\right] \mu(d \xi)<\infty
$$

Let $\sigma_{0}=\sqrt{a}$ and let $\left(\sigma_{i j}\right)$ be a $(2 \times 2)$-matrix satisfying $\left(\alpha_{i j}\right)=\left(\sigma_{i j}\right)\left(\sigma_{i j}\right)^{\tau}$. Let $(\Omega, \mathscr{F}, \mathscr{F} t, \mathbf{P})$ be a filtered probability space satisfying the usual hypotheses. Suppose that on this probability space the following adapted objects are defined:

- a 3-dimensional Brownian motion $\left\{\left(B_{0}(t), B_{1}(t), B_{2}(t)\right)\right\}$;

- a Poisson random measure $N_{0}(d s, d \xi)$ on $\mathbb{R}_{+}^{3}$ with intensity $d s m(d \xi)$;

- a Poisson random measure $N_{1}(d s, d u, d \xi)$ on $\mathbb{R}_{+}^{4}$ with intensity $d s d u \mu(d \xi)$.

We assume that $\left\{\left(B_{0}(t), B_{1}(t), B_{2}(t)\right)\right\},\left\{N_{0}(d s, d \xi)\right\}$ and $\left\{N_{1}(d s, d u, d \xi)\right\}$ are independent of each other. Let $x(0)$ and $y(0)$ be non-negative $\mathscr{F}_{0}$-measurable random variables defined on $\left(\Omega, \mathscr{F}, \mathscr{F}_{t}, \mathbf{P}\right)$. We consider the equation system

$$
\begin{aligned}
x(t)= & x(0)+\int_{0}^{t}\left(b_{1}+\beta_{11} x(s)\right) d s+\int_{0}^{t} \sigma_{11} \sqrt{2 x(s)} d B_{1}(s) \\
& +\int_{0}^{t} \sigma_{12} \sqrt{2 x(s)} d B_{2}(s)+\int_{0}^{t} \int_{\mathbb{R}_{+}^{2}} \xi_{1} N_{0}(d s, d \xi) \\
& +\int_{0}^{t} \int_{0}^{x(s-)} \int_{\mathbb{R}_{+}^{2}} \xi_{1} \tilde{N}_{1}(d s, d u, d \xi), \\
y(t)= & y(0)+\int_{0}^{t}\left(b_{2}+\beta_{21} x(s) y(s)+\beta_{22} y(s)\right) d s+\int_{0}^{t} \sigma_{0} \sqrt{2 y(s)} d B_{0}(s) \\
& +\int_{0}^{t} \sigma_{21} \sqrt{2 x(s) y(s)} d B_{1}(s)+\int_{0}^{t} \sigma_{22} \sqrt{2 x(s) y(s)} d B_{2}(s) \\
& +\int_{0}^{t} \int_{\mathbb{R}_{+}^{2}} \xi_{2} N_{0}(d s, d \xi)+\int_{0}^{t} \int_{0}^{l x(s-) y(s-)} \int_{\mathbb{R}_{+}^{2}} \xi_{2} \tilde{N}_{1}(d s, d u, d \xi) .
\end{aligned}
$$

Theorem 3.3 ([15]) The equation system given by (3.11) and (3.12) has a unique strong solution $\{(x(t), y(t))\}$. 
Following Dawson and Fleischmann [1], we call $\{(x(t), y(t))\}$ a catalytic CBI-process, where $\{(x(t))\}$ is the catalyst process and $\{y(t)\}$ is the reactant process. It is not hard to see that $\{x(t)\}$ is a CBI-process. Intuitively, we may think of $\{y(t)\}$ as a CBI-process with random branching catalysts governed by the process $\{x(t)\}$. A slightly more general catalytic CBI-process with two reactant processes was considered in [15].

\section{Two-dimensional affine processes}

The concept of affine Markov processes was introduced in the study of financial models; see, e.g., [19] and the references therein. For simplicity we only consider those processes in the twodimensional case. Let $D=\mathbb{R}_{+} \times \mathbb{R}$ and $U=\mathbb{C}_{-} \times(i \mathbb{R})$, where $\mathbb{C}_{-}=\left\{a+i b: a \in \mathbb{R}_{-}, b \in \mathbb{R}\right\}$ and $i \mathbb{R}=\{i b: b \in \mathbb{R}\}$. A transition semigroup $\left(Q_{t}\right)_{t \geq 0}$ on $D$ is called a homogeneous affine semigroup (HA-semigroup) if for each $t \geq 0$ there exists a continuous operator $u \mapsto \psi(t, u)$ on $U$ such that

$$
\int_{D} \exp \{\langle u, \xi\rangle\} Q_{t}(x, d \xi)=\exp \{\langle x, \psi(t, u)\rangle\}, \quad x \in D, u \in U .
$$

(The phrase "homogeneous affine" comes from the homogeneous affine transformation $x \mapsto$ $\langle x, \psi(t, u)\rangle$.) We say the HA-semigroup defined above is regular if it is stochastically continuous and the derivative $(\partial \psi / \partial t)(0, u)$ exists for all $u \in U$ and is continuous at $u=0$.

Clearly, the HA-semigroup satisfies the branching property (2.1), so the probability measure $Q_{t}(x, \cdot)$ is infinitely divisible. To simplify the presentation, we assume that $\left(Q_{t}\right)_{t \geq 0}$ and all probabilities on $D$ possess finite first absolute moments. Then the infinite divisibility of $Q_{t}(x, \cdot)$

and the special structure of $D$ imply that $\psi_{2}(t, u)=\beta_{22}(t) u_{2}$ for some $\beta_{22}(t) \in \mathbb{R}$ and $\psi_{1}(t, u)$ has the representation

$$
\psi_{1}(t, u)=\beta_{11}(t) u_{1}+\beta_{12}(t) u_{2}+\alpha(t) u_{2}^{2}+\int_{D}\left(e^{\langle u, \xi\rangle}-1-u_{2} \xi_{2}\right) \mu(t, d \xi),
$$

where $\alpha(t) \in \mathbb{R}_{+},\left(\beta_{11}(t), \beta_{12}(t)\right) \in D$ and $\mu(t, d \xi)$ is a $\sigma$-finite measure on $D$ supported by $D \backslash\{0\}$ such that

$$
\int_{D}\left(\left|\xi_{1}\right|+\left|\xi_{2}\right| \wedge\left|\xi_{2}\right|^{2}\right) \mu(t, d \xi)<\infty
$$

see [15]. From (4.2) and the semigroup property of $\left(Q_{t}\right)_{t \geq 0}$ it follows that

$$
\begin{aligned}
\beta_{22}(r+t) & =\beta_{22}(r) \beta_{22}(t), \\
\beta_{11}(r+t) & =\beta_{11}(r) \beta_{11}(t), \\
\beta_{12}(r+t) & =\beta_{11}(r) \beta_{12}(t)+\beta_{12}(r) \beta_{22}(t), \\
\alpha(r+t) & =\beta_{11}(r) \alpha(t)+\alpha(r) \beta_{22}^{2}(t), \\
\mu(r+t, \cdot) & =\int_{D} \mu(r, d \xi) Q_{t}(\xi, \cdot)+\beta_{11}(r) \mu(t, \cdot)
\end{aligned}
$$

for any $r, t \geq 0$.

The definition of SC-semigroups certainly applies to a HA-semigroup. It was proved in Dawson and Li [15] that if $\left(\gamma_{t}\right)_{t \geq 0}$ is a stochastically continuous SC-semigroup associated with a 
regular HA-semigroup $\left(Q_{t}\right)_{t \geq 0}$, then each $\gamma_{t}$ is an infinitely divisible probability measure. Then we have the representations

$$
\int_{D} \exp \{\langle u, \xi\rangle\} \gamma_{t}(d \xi)=\exp \{\phi(t, u)\}, \quad u \in U
$$

and

$$
\phi(t, u)=b_{1}(t) u_{1}+b_{2}(t) u_{2}+a(t) u_{2}^{2}+\int_{D}\left(e^{\langle u, \xi\rangle}-1-u_{2} \xi_{2}\right) m(t, d \xi)
$$

where $a(t) \in \mathbb{R}_{+},\left(b_{1}(t), b_{2}(t)\right) \in D$ and $m(t, d \xi)$ is a $\sigma$-finite measure on $D$ supported by $D \backslash\{0\}$ such that

$$
\int_{D}\left[\left|\xi_{1}\right|+\left|\xi_{2}\right| \wedge\left|\xi_{2}\right|^{2}\right] m(t, d \xi)<\infty
$$

Proposition 4.1 (15]) If $\left(\gamma_{t}\right)_{t \geq 0}$ is a stochastically continuous SC-semigroup given by (4.8) and (4.9), then for any $r, t \geq 0$ we have

$$
\begin{aligned}
b_{1}(r+t) & =b_{1}(r) \beta_{11}(t)+b_{1}(t) \\
b_{2}(r+t) & =b_{1}(r) \beta_{12}(t)+b_{2}(r) \beta_{22}(t)+b_{2}(t) \\
a(r+t) & =b_{1}(r) \alpha(t)+a(r) \beta_{22}^{2}(t)+a(t), \\
m(r+t, \cdot) & =\int_{D} m(r, d \xi) Q_{t}(\xi, \cdot)+b_{1}(r) \mu(t, \cdot)+m(t, \cdot) .
\end{aligned}
$$

The equations (4.10)-(4.13) give an alternative expression of the property (2.4) and make it possible to treat separately the coefficients in (4.9). This leads to some explicit analysis of the differentiability of $t \mapsto \phi(t, u)$. In particular, if $\nu$ is an infinitely divisible probability measure on $D$, we can define an SC-semigroup $\left(\gamma_{t}\right)_{t \geq 0}$ by (4.8) by letting

$$
\phi(t, u)=\int_{0}^{t} \log \hat{\nu}(\psi(s, u)) d s, \quad t \geq 0, u \in U
$$

where $\hat{\nu}$ is the characteristic function of $\nu$. In this case, we call $\left(\gamma_{t}\right)_{t \geq 0}$ a regular SC-semigroup. A simple but irregular SC-semigroup can be constructed by letting $Q_{t}$ be the identity and letting $\gamma_{t}=\delta_{\left(0, b_{2}(t)\right)}$ where $b_{2}(t)$ is a discontinuous solution of $b_{2}(r+t)=b_{2}(r)+b_{2}(t)$; see, e.g., 61, p.37]. This example shows that some condition on the function $t \mapsto b_{2}(t)$ has to be imposed to get the regularity of the SC-semigroup $\left(\gamma_{t}\right)_{t \geq 0}$ given by (4.8) and (4.9). The proof of [15. Theorem 3.1] gives the following

Theorem 4.1 ([15]) Let $\left(\gamma_{t}\right)_{t \geq 0}$ be a stochastically continuous $S C$-semigroup given by (4.8) and (4.9). Then the following conditions are equivalent:

(i) $\left(\gamma_{t}\right)_{t \geq 0}$ is regular;

(ii) $(\partial \phi / \partial t)(0, u)$ exists for every $u \in U$ and is continuous at $u=0$;

(iii) $t \mapsto b_{2}(t)$ is absolutely continuous on $[0, \infty)$. 
Suppose that $\left(Q_{t}\right)_{t \geq 0}$ is a HA-semigroup given by (4.1) and $\left(\gamma_{t}\right)_{t \geq 0}$ is an associated SCsemigroup given by (4.8) and (4.9). Let $P_{t}(x, \cdot)=Q_{t}(x, \cdot) * \gamma_{t}(\cdot)$. Then $\left(P_{t}\right)_{t \geq 0}$ is also a Markov transition semigroup on $D$ and

$$
\int_{D} \exp \{\langle u, \xi\rangle\} P_{t}(x, d \xi)=\exp \{\langle x, \psi(t, u)\rangle+\phi(t, u)\}, \quad x \in D, u \in U .
$$

In general, a Markov transition semigroup on $D$ with characteristic function of the form (4.15) is called an affine semigroup; see, e.g., 19]. We say the affine semigroup is regular if it is stochastically continuous and the derivatives $(\partial \psi / \partial t)(0, u)$ and $(\partial \phi / \partial t)(0, u)$ exist for all $u \in U$ and are continuous at $u=0$. Clearly, $\left(P_{t}\right)_{t \geq 0}$ is regular if and only if both $\left(Q_{t}\right)_{t \geq 0}$ and $\left(\gamma_{t}\right)_{t \geq 0}$ are regular. Therefore, the above theorem gives a partial answer to the open problem of characterizing all affine semigroups without the regularity assumption; see [19, Remark 2.11]. The class of regular affine semigroups was characterized completely in [19. It was shown in [15] that a regular affine process arises naturally in a limit theorem for the difference of a pair of reactant processes in a catalytic CBI-process.

\section{Measure-valued immigration processes}

Let $E$ be a Lusin topological space, i.e., a homeomorph of a Borel subset of a compact metric space. Recall that $M(E)$ is the space of finite Borel measures on $E$ endowed with the topology of weak convergence. A Markov process with state space $M(E)$ is called an $M B$-process if its transition semigroup $\left(Q_{t}\right)_{t \geq 0}$ satisfies the branching property (2.1). MB-processes appeared in Jiřina [37, 38] and Watanabe [71] as high density limits of branching particle systems. A very important special class of MB-processes, known as Dawson-Watanabe processes, have been studied extensively in the past decades; see, e.g., 9, 20, 21, 40. The development of this subject has been stimulated from different subjects including branching processes, interacting particle systems, stochastic partial differential equations and non-linear partial differential equations. The study of superprocesses has also led to better understanding of results in those subjects.

Suppose that $\left(\gamma_{t}\right)_{t \geq 0}$ is an SC-semigroup associated with $\left(Q_{t}\right)_{t \geq 0}$ and $\left(Q_{t}^{\gamma}\right)_{t \geq 0}$ is defined by (2.3). A Markov process with transition semigroup $\left(Q_{t}^{\gamma}\right)_{t \geq 0}$ is called an immigration process associated with $\left(Q_{t}\right)_{t \geq 0}$. This formulation of immigration processes was given in Li 44, 45. The intuitive meaning of the immigration model is clear from the definition of $\left(Q_{t}^{\gamma}\right)_{t \geq 0}$. Clearly, this formulation essentially includes all immigration mechanisms that are independent of the inner population.

Theorem 5.1 (44]) A family of probability measures $\left(\gamma_{t}\right)_{t \geq 0}$ on $M(E)$ is an SC-semigroup associated with $\left(Q_{t}\right)_{t \geq 0}$ if and only if there is an infinitely divisible probability entrance law $\left(K_{t}\right)_{t>0}$ for $\left(Q_{t}\right)_{t \geq 0}$ such that

$$
\log \int_{M(E)} e^{-\nu(f)} \gamma_{t}(d \nu)=\int_{0}^{t}\left[\log \int_{M(E)} e^{-\nu(f)} K_{s}(d \nu)\right] d s, \quad t \geq 0, f \in B(E)^{+} .
$$

Let us consider the case of a Dawson-Watanabe superprocess. Suppose that $\left(P_{t}\right)_{t \geq 0}$ is the transition semigroup of a Borel right process $\xi$ with state space $E$ and $\phi(\cdot, \cdot)$ is a branching mechanism given by

$$
\phi(x, z)=b(x) z+c(x) z^{2}+\int_{0}^{\infty}\left(e^{-z u}-1+z u\right) m(x, d u), \quad x \in E, z \geq 0,
$$


where $b \in B(E), c \in B(E)^{+}$and $\left(u \wedge u^{2}\right) m(x, d u)$ is a bounded kernel from $E$ to $(0, \infty)$. Then for each $f \in B(E)^{+}$the evolution equation

$$
V_{t} f(x)+\int_{0}^{t} d s \int_{E} \phi\left(y, V_{s} f(y)\right) P_{t-s}(x, d y)=P_{t} f(x), \quad t \geq 0, x \in E
$$

has a unique solution $V_{t} f \in B(E)^{+}$, and there is a Markov semigroup $\left(Q_{t}\right)_{t \geq 0}$ on $M(E)$ such that

$$
\int_{M(E)} e^{-\nu(f)} Q_{t}(\mu, d \nu)=\exp \left\{-\mu\left(V_{t} f\right)\right\}, \quad f \in B(E)^{+}
$$

Clearly, $\left(Q_{t}\right)_{t \geq 0}$ satisfies the branching property (2.1). A Markov process having transition semigroup $\left(Q_{t}\right)_{t \geq 0}$ is called a Dawson-Watanabe with parameters $(\xi, \phi)$ or simply a $(\xi, \phi)$ superprocess. This process is a natural generalization of the CB-process; see, e.g., [9]. The family of operators $\left(V_{t}\right)_{t \geq 0}$ form a semigroup, which is called the cumulant semigroup of the superprocess. Under our hypotheses, $\left(Q_{t}\right)_{t \geq 0}$ has a Borel right realization; see [22, 23].

Let $\mathscr{K}(P)$ be the set of entrance laws $\kappa=\left(\kappa_{t}\right)_{t>0}$ for the underlying semigroup $\left(P_{t}\right)_{t \geq 0}$ that satisfy $\int_{0}^{1} \kappa_{s}(E) d s<\infty$. We endow $\mathscr{K}(P)$ with the $\sigma$-algebra generated by the mappings $\kappa \mapsto \kappa_{t}(f)$ with $t>0$ and $f \in B(E)^{+}$. For $\kappa \in \mathscr{K}(P)$, set

$$
S_{t}(\kappa, f)=\kappa_{t}(f)-\int_{0}^{t} d s \int_{E} \phi\left(y, V_{s} f(y)\right) \kappa_{t-s}(d y), \quad t>0, f \in B(E)^{+} .
$$

Let $\mathscr{K}^{1}(Q)$ denote the set of probability entrance laws $K=\left(K_{t}\right)_{t>0}$ for the semigroup $\left(Q_{t}\right)_{t \geq 0}$ of the $(\xi, \phi)$-superprocess such that

$$
\int_{0}^{1} d s \int_{M(E)} \nu(E) K_{s}(d \nu)<\infty
$$

Theorem 5.2 (45]) A probability entrance law $K \in \mathscr{K}^{1}(Q)$ is infinitely divisible if and only if its Laplace functional has the representation

$$
\int_{M(E)} e^{-\nu(f)} K_{t}(d \nu)=\exp \left\{-S_{t}(\kappa, f)-\int_{\mathscr{K}(P)}\left(1-e^{-S_{t}(\eta, f)}\right) J(d \eta)\right\},
$$

where $\kappa \in \mathscr{K}(P)$ and $J$ is a $\sigma$-finite measure on $\mathscr{K}(P)$ satisfying

$$
\int_{0}^{1} d s \int_{\mathscr{K}(P)} \eta_{s}(1) J(d \eta)<\infty .
$$

The above theorem characterizes a class of infinitely divisible probability entrance laws for $\left(Q_{t}\right)_{t \geq 0}$. The right hand side of (5.6) corresponds to an infinitely divisible probability measure on $\mathscr{K}(P)$. If $\left(\kappa_{t}\right)_{t>0}$ is given by $\kappa_{t}=\mu P_{t}$, we have clearly $S_{t}(\kappa, f)=\mu\left(V_{t} f\right)$. Let $\lambda \in M(E)$ and let $L$ be a $\sigma$-finite measure on $M(E)$ satisfying

$$
\int_{M(E)} \nu(1) L(d \nu)<\infty .
$$


We can define an infinitely divisible probability entrance law $K \in \mathscr{K}^{1}(Q)$ by

$$
\int_{M(E)} e^{-\nu(f)} K_{t}(d \nu)=\exp \left\{-\lambda\left(V_{t} f\right)-\int_{M(E)}\left(1-e^{-\nu\left(V_{t} f\right)}\right) L(d \nu)\right\} .
$$

This entrance law can be closed by an infinitely divisible probability measure on $M(E)$. In this case, the transition semigroup of the corresponding immigration process is given by

$$
\begin{aligned}
\int_{M(E)} e^{-\nu(f)} Q_{t}^{\gamma}(\mu, d \nu)=\exp \{ & -\mu\left(V_{t} f\right)-\int_{0}^{t}\left[\lambda\left(V_{s} f\right)\right. \\
& \left.\left.+\int_{M(E)}\left(1-e^{-\nu\left(V_{s} f\right)}\right) L(d \nu)\right] d s\right\} .
\end{aligned}
$$

This is the case considered in $\mathrm{Li}$ [3]. It was proved in $\mathrm{Li}$ [4] following the arguments of Fitzsimmons [22, 23] that $\left(Q_{t}^{\gamma}\right)_{t \geq 0}$ is a Borel right semigroup.

Needless to say, most of the theory of Dawson-Watanabe superprocesses carries over to their associated immigration processes and could be developed by techniques very close to those of Dawson 9. However, the immigration processes have many additional structures, as might be expected from (2.3) and (2.4). A construction for the immigration processes were given in $\mathrm{Li}$ [50] by picking up measure-valued paths with random times of birth and death. The construction was based on the observation that any SC-semigroup is determined by a continuous increasing measure-valued path $\left(\eta_{t}\right)_{t \geq 0}$ and an entrance rule $\left(G_{t}\right)_{t \geq 0}$. This structure yields a natural decomposition of the immigration into two parts, the deterministic part represented by $\left(\eta_{t}\right)_{t \geq 0}$ and the random part determined by $\left(G_{t}\right)_{t \geq 0}$. The latter is an inhomogeneous immigration process and can be constructed by summing up paths $\left\{w_{t}: \alpha<t<\beta\right\}$ in the associated Kuznetsov process. By analyzing the asymptotic behavior of the paths $\left\{w_{t}: \alpha<t<\beta\right\}$ near the birth time $\alpha=\alpha(w)$, it was shown in [50] that almost all these paths start propagation in an extension of the underlying space. Those combined with the construction mentioned above give a full description of the immigration phenomenon. As an application of the construction, Li [50] gave reformulations of some well-known results on excessive measures in terms of stationary immigration superprocesses. The immigration phenomena associated with branching particle systems were studied in [46].

The state space of the immigration superprocess can be extended to include some infinite measures; see, e.g., 43. 53. With such extensions, the immigration can be governed by a $\sigma$-finite measure. A central limit theorem for the $d$-dimensional super-Brownian motion with immigration was proved in $\mathrm{Li}$ and Shiga [52], where the immigration is governed by a deterministic $\sigma$-finite measure. When the governing measure is the Lebesgue measure, the normalization function is $t^{3 / 4}$ for $d=1,(t \log t)^{1 / 2}$ for $d=2$ and $t^{1 / 2}$ for $d \geq 3$. The corresponding large deviation principle was obtained in Zhang [72] with the normalization function $t$ in all dimensions and the speed function $t^{1 / 2}$ for $d=1, t / \log t$ for $d=2$ and $t$ for $d \geq 3$; see also [75]. The gap between the central limit theorem and the large deviation principle was filled in Zhang [73. by establishing a moderate deviation principle. More precisely, she proved that this immigration superprocess satisfies a large deviations principle under the normalization $t^{1-\delta / 4}$ for $d=1$, $t^{1-\delta / 2}(\log t)^{\delta / 2}$ for $d=2$ and $t^{1-\delta / 2}$ for $d \geq 3$, where $\delta \in(0,1)$ is a parameter; see also [35, 74].

A super-Brownian motion with immigration governed by another super-Brownian was introduced and studied in Hong and Li [34. They established a central limit theorem for the process 
which leads to Gaussian random fields in high dimensions. For $d=3$ the field is spatially uniform, for $d \geq 5$ its covariance is given by the potential operator of the underlying Brownian motion and for $d=4$ it involves a mixture of the two kinds of fluctuations, which seems to be a new phenomenon in the asymptotic behavior of measure-valued processes. There is a similar phenomenon in the central limit theorem of the corresponding occupation times obtained in Hong [30] with $d=6$ being critical. Some quenched mean limit theorems were proved in Hong 33. The moderate deviation principles for the immigration superprocesses were established in 31]. Large deviation problems were studied in 32, where the speed functions are $t^{1 / 2}$ in $d=3$ and $t$ in $d \geq 4$. For $d \neq 4$ the principle was accomplished by the well-known Gärtner-Ellis theorem. In the critical dimension $d=4$, the large deviation problem is much more difficult and only the limit superior was established. We refer the reader to [53] for a more detailed survey on the early results measure-valued immigration processes.

\section{Excursions and generalized immigration processes}

Let $\alpha>0$ be a constant and $\{B(t): t \geq 0\}$ a standard Brownian motion. For any initial condition $x(0)=x \geq 0$ the stochastic differential equation

$$
d x(t)=\sqrt{2 \alpha x(t)} d B(t), \quad t \geq 0
$$

has a unique non-negative solution $\{x(t): t \geq 0\}$, which is a special case of the CB-process. This process is known as a Feller branching diffusion in the literature. The transition semigroup $\left(Q_{t}\right)_{t \geq 0}$ of the process is determined by

$$
\int_{0}^{\infty} e^{-z y} Q_{t}(x, d y)=\exp \left\{-x z(1+\alpha t z)^{-1}\right\}, \quad t, x, z \geq 0
$$

see, e.g., [36, p.236]. In view of the infinite divisibility implied by (6.2), there is a family of canonical measures $\left(\kappa_{t}\right)_{t>0}$ on $(0, \infty)$ such that

$$
\int_{0}^{\infty}\left(1-e^{-z y}\right) \kappa_{t}(d y)=z(1+\alpha t z / 2)^{-1}, \quad t>0, z \geq 0 .
$$

Indeed, it is easy to check that

$$
\kappa_{t}(d y)=(\alpha t)^{-2} e^{-y / \alpha t} d y, \quad t, x>0 .
$$

Let $Q_{t}^{\circ}(x, d y)$ denote the restriction to $(0, \infty)$ of the kernel $Q_{t}(x, d y)$. Since zero is a trap for the Feller branching diffusion, $\left(Q_{t}^{\circ}\right)_{t \geq 0}$ also constitute a semigroup. Based on (6.2) and (6.3) it is not hard to show that $\kappa_{r} Q_{t}^{\circ}=\kappa_{r+t}$ for all $r, t>0$. In other words, $\left(\kappa_{t}\right)_{t>0}$ is an entrance law for $\left(Q_{t}^{\circ}\right)_{t \geq 0}$.

Let $W=C\left([0, \infty), \mathbb{R}_{+}\right)$and let $\tau_{0}(w)=\inf \left\{s>0: w_{s}=0\right\}$ for $w \in W$. Let $W_{0}$ be the set of paths $w \in W$ such that $w_{0}=w_{t}=0$ for $t \geq \tau_{0}(w)$. We endow $W$ and $W_{0}$ with the topology of locally uniform convergence. By the theory of Markov processes, there is a unique $\sigma$-finite measure $\mathbf{Q}_{\kappa}$ on $\left(W_{0}, \mathscr{B}\left(W_{0}\right)\right)$ such that

$$
\mathbf{Q}_{\kappa}\left\{w_{t_{1}} \in d y_{1}, \cdots, w_{t_{n}} \in d y_{n}\right\}=\kappa_{t_{1}}\left(d y_{1}\right) Q_{t_{2}-t_{1}}^{\circ}\left(y_{1}, d y_{2}\right) \cdots Q_{t_{n}-t_{n-1}}^{\circ}\left(y_{n-1}, d y_{n}\right)
$$


for $0<t_{1}<t_{2}<\cdots<t_{n}$ and $y_{1}, y_{2}, \cdots, y_{n} \in(0, \infty)$; see, e.g., [59]. The measure $\mathbf{Q}_{\kappa}$ is known as the excursion law of the Feller branching diffusion. Roughly speaking, (6.5) asserts that $\left\{w_{t}: t>0\right\}$ under $\mathbf{Q}_{\kappa}$ is a Feller branching diffusion with one-dimensional distributions $\left\{\kappa_{t}: t>0\right\}$. The Feller branching diffusion can be reconstructed from the excursion law $\mathbf{Q}_{\kappa}$ in the following way: Fix $x \geq 0$ and let $N(d w)$ be a Poisson random measure on $W_{0}$ with intensity $x \mathbf{Q}_{\kappa}(d w)$. Let $x(0)=x$ and

$$
x(t)=\int_{W_{0}} w_{t} N(d w), \quad t>0 .
$$

Then $\{x(t): t \geq 0\}$ is a weak solution of (6.1); see [59, Theorem 4.1].

Let $b(\cdot)$ be a non-negative and locally Lipschitz function on $\mathbb{R}_{+}$satisfying the linear growth condition. A non-negative diffusion process $\{y(t): t \geq 0\}$ can be defined by the stochastic differential equation

$$
d y(t)=\sqrt{2 \alpha y(t)} d B(t)+b(y(t)) d t, \quad t \geq 0 .
$$

This process can be constructed from a Feller branching diffusion and a Poisson random measure based on $\mathbf{Q}_{\kappa}(d w)$ as follows. Let $\{x(t): t \geq 0\}$ be a Feller branching diffusion and let $N(d s, d u, d w)$ be a Poisson random measure on $\mathbb{R}_{+}^{2} \times W_{0}$ with intensity $d s d u \mathbf{Q}_{\kappa}(d w)$. We assume that $\{x(t): t \geq 0\}$ and $\{N(d s, d u, d w)\}$ are independent.

Proposition 6.1 (24]) There is a unique strong solution of the stochastic equation

$$
y(t)=x(t)+\int_{0}^{t} \int_{0}^{b(y(s))} \int_{W_{0}} w_{t-s} N(d s, d u, d w), \quad t \geq 0 .
$$

Moreover, the solution $\{y(t): t \geq 0\}$ of the above equation is a weak solution of (6.7).

This proposition is a consequence of $\mathrm{Fu}$ and $\mathrm{Li}$ [24, Theorem 4.1], where more general results on measure-valued processes were given. In particular, if $b(x) \equiv b$ is a constant, $\{y(t): t \geq 0\}$ is a CBI-process associated with the Feller branching diffusion; see [59]. In the general case, we may regard $\{y(t): t \geq 0\}$ as a generalized CBI-process.

The approach of stochastic equations driven by Poisson random measures based on the excursion law has more substantial applications in constructions of some measure-valued diffusions. Let us look at an example of this type involving a stochastic flow. Suppose that $h$ is a continuously differentiable function on $\mathbb{R}$ such that both $h$ and $h^{\prime}$ are square-integrable. Then the function

$$
\rho(x)=\int_{\mathbb{R}} h(y-x) h(y) d y, \quad x \in \mathbb{R}
$$

is twice continuously differentiable with bounded derivatives $\rho^{\prime}$ and $\rho^{\prime \prime}$. Let $m$ be a $\sigma$-finite Borel measure on $\mathbb{R}$ and $q(\cdot, \cdot)$ a non-negative Borel function on $M(\mathbb{R}) \times \mathbb{R}$ such that there is a constant $K$ such that

$$
\int_{\mathbb{R}} q(\mu, y) m(d y) \leq K(1+\|\mu\|), \quad \mu \in M(\mathbb{R}),
$$


and for each $R>0$ there is a constant $K_{R}>0$ such that

$$
\int_{\mathbb{R}}|q(\mu, y)-q(\nu, y)| m(d y) \leq K_{R}\|\mu-\nu\|
$$

for $\mu$ and $\nu \in M(\mathbb{R})$ satisfying $\mu(\mathbb{R}) \leq R$ and $\nu(\mathbb{R}) \leq R$, where $\|\cdot\|$ denotes the total variation of the signed measure. Let us consider the following martingale problem of an $M(\mathbb{R})$-valued process $\left\{Y_{t}: t \geq 0\right\}$ : For each $\phi \in C^{2}(\mathbb{R})$,

$$
M_{t}(\phi):=Y_{t}(\phi)-Y_{0}(\phi)-\rho(0) \int_{0}^{t} Y_{s}\left(\phi^{\prime \prime}\right) d s-\int_{0}^{t} d s \int_{\mathbb{R}} \phi(y) q\left(Y_{s}, y\right) m(d y)
$$

is a continuous martingale with quadratic variation process

$$
\langle M(\phi)\rangle_{t}=2 \alpha \int_{0}^{t} Y_{s}\left(\phi^{2}\right) d s+\int_{0}^{t} d s \int_{\mathbb{R}^{2}} \rho(x-y) \phi^{\prime}(x) \phi^{\prime}(y) Y_{s}^{2}(d x, d y) .
$$

Let $W(d t, d y)$ be a time-space white noise on $[0, \infty) \times \mathbb{R}$ based on the Lebesgue measure; see, e.g., 66]. By [17, Lemma 3.1] or [70, Lemma 1.3], for any $r \geq 0$ and $a \in \mathbb{R}$ the stochastic equation

$$
x(t)=a+\int_{r}^{t} \int_{\mathbb{R}} h(y-x(s)) W(d s, d y), \quad t \geq r
$$

has a unique continuous solution $\{x(r, a, t): t \geq r\}$, which is a Brownian motion with quadratic variation $\rho(0) d t$. Indeed, the system $\{x(r, a, t): t \geq r ; a \in \mathbb{R}\}$ determines an isotropic stochastic flow. Fix $\mu \in M(\mathbb{R})$ and let $N_{0}(d a, d w)$ be a Poisson random measure on $\mathbb{R} \times W_{0}$ with intensity $\mu(d a) \mathbf{Q}_{\kappa}(d w)$ and $N(d s, d a, d u, d w)$ a Poisson random measure on $\mathbb{R}_{+} \times \mathbb{R} \times \mathbb{R}_{+} \times W_{0}$ with intensity $d s m(d a) d u \mathbf{Q}_{\kappa}(d w)$. Suppose that $\{W(d t, d y)\},\left\{N_{0}(d a, d w)\right\}$ and $\{N(d s, d a, d u, d w)\}$ are independent of each other.

Theorem 6.1 (13]) There is a unique strong solution of the stochastic equation

$$
\begin{aligned}
Y_{t}= & \int_{\mathbb{R}} \int_{W_{0}} w(t) \delta_{x(0, a, t)} N_{0}(d a, d w) \\
& +\int_{0}^{t} \int_{\mathbb{R}} \int_{0}^{q\left(Y_{s}, a\right)} \int_{W_{0}} w(t-s) \delta_{x(s, a, t)} N(d s, d a, d u, d w), \quad t>0 .
\end{aligned}
$$

Furthermore, if we set $Y_{0}=\mu$, the process $\left\{Y_{t}: t \geq 0\right\}$ is a measure-valued diffusion process solving the martingale problem given by (6.12) and (6.13).

In view of [6.15), we may regard $\left\{Y_{t}: t \geq 0\right\}$ as a generalized immigration superprocess carried by the stochastic flow given by (6.14). The stochastic equation (6.15) is substantial for the construction of this measure-valued diffusion process, for the uniqueness of solution of the martingale problem given by (6.12) and (6.13) still remains open. 


\section{Generalized Mehler semigroups}

Let $H$ be a real separable Hilbert space and let $\left(T_{t}\right)_{t \geq 0}$ be a strongly continuous semigroup of linear operators on $H$. A family of probability measures $\left(\gamma_{t}\right)_{t \geq 0}$ on $H$ is called an $S C$-semigroup associated with $\left(T_{t}\right)_{t \geq 0}$ if it satisfies

$$
\gamma_{r+t}=\left(\gamma_{r} \circ T_{t}^{-1}\right) * \gamma_{t}, \quad r, t \geq 0 .
$$

This is clearly the special case of (2.4) with $Q_{t}(x, \cdot) \equiv \delta_{T_{t} x}$. If (17.1) is satisfied, we can define a Markov transition semigroup $\left(Q_{t}^{\gamma}\right)_{t \geq 0}$ on $H$ by

$$
Q_{t}^{\gamma} f(x)=\int_{H} f\left(T_{t} x+y\right) \mu_{t}(d y), \quad x \in H, f \in B(H),
$$

which is called a generalized Mehler semigroup associated with $\left(T_{t}\right)_{t>0}$. The corresponding Markov process is a generalized OU-process; see [4.

According to a result of Schmuland and Sun [62, if $\left(\gamma_{t}\right)_{t \geq 0}$ is a solution of (7.1), each $\gamma_{t}$ is an infinitely divisible probability measure. By Linde [55, p.75 and p.84], we have the following representation of the characteristic functional:

$$
\begin{aligned}
\hat{\gamma}_{t}(a)=\exp \{ & \left\{\left\langle b_{t}, a\right\rangle-\frac{1}{2}\left\langle R_{t} a, a\right\rangle\right. \\
& \left.+\int_{H^{\circ}}\left(e^{i\langle x, a\rangle}-1-i\langle x, a\rangle 1_{[0,1]}(\|x\|)\right) M_{t}(d x)\right\}, \quad t \geq 0, a \in H,
\end{aligned}
$$

where $b_{t} \in H, R_{t}$ is a symmetric, positive-definite nuclear operator on $H$, and $M_{t}$ is a $\sigma$-finite measure (Lévy measure) on $H^{\circ}:=H \backslash\{0\}$ satisfying

$$
\int_{H^{\circ}}\left(1 \wedge\|x\|^{2}\right) M_{t}(d x)<\infty
$$

Theorem 7.1 (16]) Suppose that $\left(\gamma_{t}\right)_{t \geq 0}$ is a family of probability measures on $H$. If there is a family of infinitely divisible probabilities $\left(\nu_{s}\right)_{s>0}$ such that $\nu_{r+t}=\nu_{r} \circ T_{t}^{-1}$ for all $r, t>0$ and

$$
\hat{\gamma}_{t}(a)=\exp \left\{\int_{0}^{t} \log \hat{\nu}_{s}(a) d s\right\}, \quad t \geq 0, a \in H
$$

then $\left(\gamma_{t}\right)_{t \geq 0}$ is an SC-semigroup. Conversely, if $\left(\gamma_{t}\right)_{t \geq 0}$ is an SC-semigroup given by (7.3) and if $t \mapsto b_{t}$ is absolutely continuous, then the characteristic functional $\hat{\gamma}_{t}$ has representation (7.4).

The above theorem gives a characterization for the generalized Mehler semigroup $\left(Q_{t}^{\gamma}\right)_{t \geq 0}$. If there is an infinitely divisible probability measure $\nu_{0}$ on $H$ such that $\nu_{t}=\nu_{0} \circ T_{t}^{-1}$, we say that $\left(\gamma_{t}\right)_{t \geq 0}$ and $\left(Q_{t}^{\gamma}\right)_{t \geq 0}$ are regular. In this case, the function $t \mapsto \hat{\gamma}_{t}(a)$ is differentiable for every $a \in H$. It was proved in Bogachev et al. 4] that a cylindrical Gaussian SC-semigroup satisfying this differentiability condition can be extended into a real Gaussian SC-semigroup in an enlargement of $H$ and the corresponding OU-process can be constructed as the strong solution to a stochastic differential equation. Those results were extended to the general non-Gaussian case in [25]. A simple and nice necessary and sufficient condition for the differentiability of 
$t \mapsto \hat{\gamma}_{t}(a)$ was given by van Neerven [56]. Some powerful inequalities for regular generalized Mehler semigroups were proved in Röckner and Wang [60] and Wang [67.

It was observed in Dawson et al. [16] that the OU-processes corresponding to an irregular generalized Mehler semigroup usually have no right continuous realizations. Under the second moment assumption, Dawson and Li [14] studied the construction of OU-processes corresponding to centered but irregular SC-semigroups. Based on Theorem 7.1 they showed that each centered SC-semigroup is uniquely determined by an infinitely divisible probability measure on the entrance space $\tilde{H}$ for the semigroup $\left(T_{t}\right)_{t \geq 0}$, which is an enlargement of $H$. They proved that a centered SC-semigroup can always be extended to a regular one on the entrance space. Those results provide an approach to the study of irregular generalized Mehler semigroups with which one can reduce some of their analysis to the framework of [4, 25, 67.

\section{Fluctuation limits of immigration processes}

Fluctuation limits of branching particle systems and superprocesses have been studied extensively. Since those systems are usually unstable, in many cases one uses time-dependent scalings which lead to time-inhomogeneous OU-processes; see, e.g., [5, 12] and the references therein. For subcritical branching systems with immigration, it is usually easy to find a stationary distribution. In the study of fluctuation limits of those systems, we can use a time-independent scaling, which lead to homogeneous OU-processes. Fluctuation limits of this kind were studied in [26, 27, 47, 49, 54].

Let $A_{0}$ be the generator of a conservative Feller transition semigroup on $\mathbb{R}^{d}$ such that $C^{2}\left(\mathbb{R}^{d}\right) \subseteq \mathscr{D}\left(A_{0}\right)$ and $A_{0} f \in C\left(\mathbb{R}^{d}\right)$ for every $f \in C^{2}\left(\mathbb{R}^{d}\right)$. We fix a strictly positive function $b(\cdot) \in C\left(\mathbb{R}^{d}\right)^{+}$which is bounded away from zero. Let $\left(P_{t}\right)_{t \geq 0}$ be the semigroup generated by $A:=A_{0}-b$ and let $\phi_{0}$ be a continuous function given by

$$
\phi_{0}(x, z)=c(x) z^{2}+\int_{0}^{\infty}\left(e^{-z u}-1+z u\right) n(x, d u), \quad x \in \mathbb{R}^{d}, z \geq 0,
$$

where $c(\cdot) \in C\left(\mathbb{R}^{d}\right)^{+}$and $u^{2} n(x, d u)$ is a bounded kernel from $\mathbb{R}^{d}$ to $(0, \infty)$. Then the evolution equation

$$
V_{t} f(x)+\int_{0}^{t} d s \int_{\mathbb{R}^{d}} \phi_{0}\left(y, V_{s} f(y)\right) P_{t-s}(x, d y)=P_{t} f(x), \quad t \geq 0, x \in \mathbb{R}^{d}
$$

defines a cumulant semigroup $\left(V_{t}\right)_{t \geq 0}$. Given $m \in M\left(\mathbb{R}^{d}\right)$, we can define the transition semigroup $\left(Q_{t}^{m}\right)_{t \geq 0}$ of an immigration superprocess with state space $M\left(\mathbb{R}^{d}\right)$ by

$$
\int_{M\left(\mathbb{R}^{d}\right)} e^{-\nu(f)} Q_{t}^{m}(\mu, d \nu)=\exp \left\{-\mu\left(V_{t} f\right)-\int_{0}^{t} m\left(V_{s} f\right) d s\right\}, \quad f \in C\left(\mathbb{R}^{d}\right)^{+} .
$$

Since $\left(P_{t}\right)_{t \geq 0}$ is a Feller semigroup, the immigration superprocess has a Hunt realization. In particular, it has a càdlàg realization; see, e.g., [64, p.221].

It is easy to see that $Q_{t}^{m}(\mu, \cdot)$ converges as $t \rightarrow \infty$ to the probability measure $Q_{\infty}(\cdot)$ on $M\left(\mathbb{R}^{d}\right)$ given by

$$
\int_{M\left(\mathbb{R}^{d}\right)} e^{-\nu(f)} Q_{\infty}^{m}(d \nu)=\exp \left\{-\int_{0}^{\infty} m\left(V_{s} f\right) d s\right\}, \quad f \in C\left(\mathbb{R}^{d}\right)^{+} .
$$


Clearly, $Q_{\infty}(\cdot)$ is the unique equilibrium of the semigroup $\left(Q_{t}^{m}\right)_{t \geq 0}$. Moreover, we have

$$
\int_{M\left(\mathbb{R}^{d}\right)} \nu(f) Q_{\infty}^{m}(d \nu)=\lambda(f), \quad f \in C\left(\mathbb{R}^{d}\right)^{+}
$$

where $\lambda \in M\left(\mathbb{R}^{d}\right)$ is defined by

$$
\lambda=\int_{0}^{\infty} m P_{s} d s
$$

It is a natural problem to investigate the asymptotic fluctuation of the immigration superprocess around the long-term average $\lambda$ as the branching mechanism $\phi_{0}$ decreases to zero. A result of this type is formulated as follows. For any integer $k \geq 1$ let $\phi_{k}(x, z)=\phi_{0}(x, z / k)$. Then $\phi_{k}(x, z) \rightarrow 0$ as $k \rightarrow \infty$. Suppose that $\left\{Y_{t}^{(k)}: t \geq 0\right\}$ is a càdlàg immigration superprocess with parameters $\left(A, \phi_{k}, m\right)$ and $Y_{0}^{(k)}=\lambda$. Let

$$
Z_{t}^{(k)}=k\left[Y_{t}^{(k)}-\lambda\right], \quad t \geq 0
$$

Let $\mathscr{S}\left(\mathbb{R}^{d}\right)$ denote the Schwartz space of rapidly decreasing functions on $\mathbb{R}^{d}$. That is, each $f \in \mathscr{S}\left(\mathbb{R}^{d}\right)$ is belong to $C^{\infty}\left(\mathbb{R}^{d}\right)$ and for each integer $n \geq 1$ and each non-negative integervalued vector $\alpha=\left(\alpha_{1}, \cdots, \alpha_{d}\right)$ we have

$$
\lim _{|x| \rightarrow \infty}|x|^{n}\left|\partial^{\alpha} f(x)\right|=0
$$

where

$$
\partial^{\alpha} f(x)=\frac{\partial^{|\alpha|}}{\partial x_{1}^{\alpha_{1}} \cdots x_{d}^{\alpha_{d}}} f\left(x_{1}, \cdots, x_{d}\right)
$$

and $|\alpha|=\alpha_{1}+\cdots+\alpha_{d}$. The topology of $\mathscr{S}\left(\mathbb{R}^{d}\right)$ is defined by the sequence of semi-norms

$$
f \mapsto p_{n}(f):=\sup \left\{\left(1+|x|^{n}\right)\left|\partial^{\alpha} f(x)\right|: x \in \mathbb{R}^{d},|\alpha| \leq n\right\}, \quad n=0,1,2, \cdots .
$$

Let $\mathscr{S}^{\prime}\left(\mathbb{R}^{d}\right)$ denote the dual space of $\mathscr{S}\left(\mathbb{R}^{d}\right)$ equipped with the strong topology. Then both $\mathscr{S}\left(\mathbb{R}^{d}\right)$ and $\mathscr{S}^{\prime}\left(\mathbb{R}^{d}\right)$ are nuclear spaces; see, e.g., [63 p.107]. It is easy to see that $\left\{Z_{t}^{(k)}: t \geq 0\right\}$ has sample paths in $D\left([0, \infty), \mathscr{S}^{\prime}\left(\mathbb{R}^{d}\right)\right)$.

Theorem 8.1 (26]) As $k \rightarrow \infty$, the finite dimensional distributions of $\left\{Z_{t}^{(k)}: t \geq 0\right\}$ converge to those of the $\mathscr{S}^{\prime}\left(\mathbb{R}^{d}\right)$-valued Markov process $\left\{Z_{t}: t \geq 0\right\}$ with $Z_{0}=0$ and with transition semigroup $\left(T_{t}\right)_{t \geq 0}$ defined by

$$
\int_{\mathscr{S}^{\prime}\left(\mathbb{R}^{d}\right)} e^{i\langle\nu, f\rangle} T_{t}(\mu, d \nu)=\exp \left\{i\left\langle\mu, P_{t} f\right\rangle+\int_{0}^{t} \lambda\left(\phi_{0}\left(-i P_{s} f\right)\right) d s\right\}, \quad f \in \mathscr{S}\left(\mathbb{R}^{d}\right),
$$

where $\phi_{0}\left(-i P_{s} f\right)$ is given by (8.1) with $z$ replaced by $-i P_{s} f(x)$.

The above theorem was improved in [54, where it was proved that $\left\{Z_{t}^{(k)}: t \geq 0\right\}$ converges to $\left\{Z_{t}: t \geq 0\right\}$ weakly in $D\left([0, \infty), \mathscr{S}^{\prime}\left(\mathbb{R}^{d}\right)\right)$. Indeed, the fluctuation limit theorem was also formulated in [54] in a suitable Sobolev space. For any integer $n \geq 0$ we define the Sobolev space

$$
H^{n}\left(\mathbb{R}^{d}\right)=\left\{f \in \mathscr{S}^{\prime}\left(\mathbb{R}^{d}\right): \partial^{\alpha} f \in L^{2}\left(\mathbb{R}^{d}\right) \text { whenever }|\alpha| \leq n\right\}
$$


with the norm $\|\cdot\|_{n}$ defined by

$$
\|f\|_{n}^{2}=\sum_{|\alpha| \leq n} \int_{\mathbb{R}^{d}}\left|\partial^{\alpha} f(x)\right|^{2} d x
$$

Let $H^{-n}\left(\mathbb{R}^{d}\right)$ be the strong topological dual of $H^{n}\left(\mathbb{R}^{d}\right)$. It is well-known that $H^{-n}\left(\mathbb{R}^{d}\right)$ can be identified as a subspace of $\mathscr{S}^{\prime}\left(\mathbb{R}^{d}\right)$ and

$$
\mathscr{S}^{\prime}\left(\mathbb{R}^{d}\right) \supseteq H^{m}\left(\mathbb{R}^{d}\right) \supseteq H^{n}\left(\mathbb{R}^{d}\right) \supseteq \mathscr{S}\left(\mathbb{R}^{d}\right)
$$

for any integers $m \leq n$ with continuous embeddings; see, e.g., [2, Theorem 5.5]. Now we have

Theorem 8.2 ([54]) For any integer $n>d+2$ the process $\left\{Z_{t}: t \geq 0\right\}$ has a realization in $D\left([0, \infty), H^{-n}\left(\mathbb{R}^{d}\right)\right)$ and $\left\{Z_{t}^{(k)}: t \geq 0\right\}$ converges weakly to $\left\{Z_{t}: t \geq 0\right\}$ in $D\left([0, \infty), H^{-n}\left(\mathbb{R}^{d}\right)\right)$.

By the above theorem, $\left\{Z_{t}: t \geq 0\right\}$ is a generalized OU-process in the real separable Hilbert space $H^{-n}\left(\mathbb{R}^{d}\right)$. This puts the process into the framework of generalized Mehler semigroup of the last section and makes it possible to derive regularities and properties of the processes from the existing literature; see, e.g., 4, 16, 25, 60, 67.

The limiting generalized OU-process obtained in above can live in a much smaller state space. Let us consider the case where $A_{0}=\Delta$ and $\phi_{0}(x, z)=c(x) z^{2} / 2$. In this case, the corresponding generalized OU-process solves the Langevin equation

$$
d Z_{t}=d W_{t}+\Delta Z_{t} d t-b Z_{t} d t, \quad t \geq 0,
$$

where $\left\{W_{t}: t \geq 0\right\}$ is a time-space white noise with intensity $c(x) d t \lambda(d x)$; see, e.g., 47]. Given $Z_{0}$ the solution of (8.9) is represented by

$$
Z_{t}=Z_{0} P_{t}+\int_{0}^{t} \int_{\mathbb{R}^{d}} p_{t-s}(x, \cdot) W(d s, d x), \quad t \geq 0
$$

where $p_{t}(x, \cdot)$ denotes the density of $P_{t}(x, \cdot)$. If $d=1$, the process $\left\{Z_{t}: t \geq 0\right\}$ has a version in $L^{2}(\mathbb{R})$. Indeed, it is well-known that $Z_{0} P_{t} \in L^{2}(\mathbb{R})$ whenever $Z_{0} \in L^{2}(\mathbb{R})$. On the other hand, we have

$$
\begin{aligned}
& \mathbf{E}\left[\int_{\mathbb{R}}\left(\int_{0}^{t} \int_{\mathbb{R}} p_{t-s}(x, y) W(d s, d x)\right)^{2} d y\right] \\
= & \int_{\mathbb{R}} d y \int_{0}^{t} d s \int_{\mathbb{R}} p_{t-s}(x, y)^{2} c(x) \lambda(d x) \\
\leq & \int_{0}^{t} \frac{1}{\sqrt{2 \pi(t-s)}} d s \int_{\mathbb{R}} c(x) \lambda(d x) \\
< & \infty
\end{aligned}
$$

Then the second term on the right hand side of (8.10) exists almost surely in $L^{2}(\mathbb{R})$. It follows that

$$
\int_{L^{2}(\mathbb{R})} e^{i\langle\nu, f\rangle} T_{t}(\mu, d \nu)=\exp \left\{i\left\langle\mu, P_{t} f\right\rangle-\int_{0}^{t} \lambda\left(c\left|P_{s} f\right|^{2}\right) d s\right\}, \quad f \in L^{2}(\mathbb{R})
$$


defines a generalized Mehler semigroup $\left(T_{t}\right)_{t>0}$ on $L^{2}(\mathbb{R})$. This semigroup is clearly irregular on the state space $L^{2}(\mathbb{R})$, but the characteristic functional of the corresponding SC-semigroup is differentiable in time. Measure-valued catalysts for superprocess were introduced by Dawson and Fleischmann [10]. One may also study fluctuation limits of immigration superprocesses with measure-valued catalysts. In such case the resulting SC-semigroup may have non-differentiable characteristic functionals; see [16].

Acknowledgements. This work was supported by the Creative Research Group Fund of the National Natural Science Foundation of China under grant number 10121101. I own special thanks to Professors M.F. Chen and F.Y. Wang who suggested that I write this survey. I would also like to thank Professors W.M. Hong and M. Zhang for helpful discussions on related topics.

\section{References}

[1] Athreya, K.B. and Ney, P.E.: Branching Processes. Springer-Verlag, New York (1972).

[2] Barros-Neto, J.: An introduction to the theory of distributions. Marcel Dekker, New York (1973). Chinese Translation (1981).

[3] Bertoin, J. (1996): Lévy Processes. Cambridge University Press, Cambridge.

[4] Bogachev, V.I.; Röckner, M. and Schmuland, B.: Generalized Mehler semigroups and applications. Probab. Th. Rel. Fields 105 (1996), 193-225.

[5] Bojdecki, T. and Gorostiza, L.G.: Langevin equation for $\mathscr{S}^{\prime}$-valued Gaussian processes and fluctuation limits of infinite particle systems. Probab. Th. Rel. Fields $\mathbf{7 3}$ (1986), 227-244.

[6] Chen, M.F.: Ergodic convergence rates of Markov processes - eigenvalues, inequalities and ergodic theory. In: Proceedings of "ICM 2002", Vol. III, 25-40. Higher Education Press, Beijing (2002).

[7] Chen, M.F.: From Markov Chains to Non-Equilibrium Particle Systems. Second Edition. World Scientific, Singapore (2004).

[8] Chen, M.F.: Eigenvalues, Inequalities and Ergodic Theory. Springer-Verlag, London (2004).

[9] Dawson, D.A.: Measure-Valued Markov Processes. In: Lecture Notes Math. 1541 (1993), 1-260. Springer-Verlag, New York.

[10] Dawson, D.A. and Fleischmann, K.: Critical branching in a highly fluctuating random medium. Probab. Th. Rel. Fields 90 (1991), 241-274.

[11] Dawson, D.A. and Fleischmann, K.: A continuous super-Brownian motion in a super-Brownian medium. J. Theoret. Probab. 10 (1997), 213-276.

[12] Dawson, D.A.; Fleischmann, K. and Gorostiza, L.G: Stable hydrodynamic limit fluctuations of a critical branching particle system in a random medium. Ann. Probab. 17 (1989), 1083-1117.

[13] Dawson, D.A. and Li, Z.H.: Construction of immigration superprocesses with dependent spatial motion from one-dimensional excursions. Probab. Th. Rel. Fields 127 (2003), 37-61.

[14] Dawson, D.A. and Li, Z.H.: Non-differentiable skew convolution semigroups and related OrnsteinUhlenbeck processes. Potential Anal. 20 (2004), 285-302. 
[15] Dawson, D.A. and Li, Z.H.: Skew convolution semigroups and affine Markov processes. Ann. Probab. To appear (2006). [Preprint form: math.bnu.edu.cn/ ${ }^{1 i z h}$ ]

[16] Dawson, D.A.; Li, Z.H.; Schmuland, B. and Sun, W.: Generalized Mehler semigroups and catalytic branching processes with immigration. Potential Anal. 21 (2004), 75-97.

[17] Dawson, D.A.; Li, Z.H. and Wang, H.: Superprocesses with dependent spatial motion and general branching densities. Elect. J. Probab. 6 (2001), Paper No. 25, 1-33.

[18] Dellacherie, C. and Meyer, P.A.: Probabilites and Potential. Chapters V-VIII. North-Holland, Amsterdam (1982).

[19] Duffie, D.; Filipović, D. and Schachermayer, W.: Affine processes and applications in finance. Ann. Appl. Probab. 13 (2003), 984-1053.

[20] Dynkin, E.B.: Diffusions, Superdiffusions and Partial Differential Equations. Amer. Math. Soc., Providence, RI (2002).

[21] Etheridge, A.M.: An Introduction to Superprocesses. Amer. Math. Soc., Providence, RI (2000).

[22] Fitzsimmons, P.J.: Construction and regularity of measure-valued Markov branching processes. Israel J. Math. 64 (1988), 337-361.

[23] Fitzsimmons, P.J.: On the martingale problem for measure-valued Markov branching processes. In: Seminar on Stochastic Processes 1991 (1992), 39-51. Birkhäuser Boston, Inc., Boston, MA.

[24] Fu, Z.F. and Li, Z.H.: Measure-valued diffusions and stochastic equations with Poisson process. Osaka J. Math. 41 (2004), 727-744.

[25] Fuhrman, M. and Röckner, M.: Generalized Mehler semigroups: The non-Gaussian case. Potential Anal. 12 (2000), 1-47.

[26] Gorostiza, L.G. and Li, Z.H.: Fluctuation limits of measure-valued immigration processes with small branching. In: Aportaciones Matemáticas: Investigación 14, 261-268. Sociedad Matemática Mexicana.

[27] Gorostiza, L.G. and Li, Z.H.: High density fluctuations of immigration branching particle systems. In: CMS Conference Proceedings. Series 26 (2000), 159-171. Amer. Math. Soc., Providence, RI.

[28] Grey, D.R.: Asymptotic behavior of continuous time, continuous state-space branching processes. J. Appl. Probab. 11 (1974), 669-677.

[29] Harris, T.E.: The Theory of Branching Processes. Springer-Verlag, Berlin (1963).

[30] Hong, W.M.: Longtime behavior for the occupation time processes of a super-Brownian motion with random immigration. Stochastic Process. Appl. 102 (2002), 43-62.

[31] Hong, W.M.: Moderate deviation for the super-Brownian motion with super-Brownian immigration. J. Applied Probab. 39 (2002), 829-838.

[32] Hong, W.M.: Large deviations for the super-Brownian motion with super-Brownian immigration. J. Theoret. Probab. 16 (2003), 899-922.

[33] Hong, W.M.: Quenched mean limit theorems for the super-Brownian motion with super-Brownian immigration. Infin. Dimens. Anal. Quantum Probab. Relat. Top. 8 (2005), 383-396. 
[34] Hong, W.M. and Li, Z.H.: A central limit theorem for super Brownian motion with super Brownian immigration. J. Appl. Probab. 36 (1999), 1218-1224.

[35] Hong, W.M. and Li, Z.H.: Large and moderate deviations for occupation times of immigration superprocesses. Infin. Dimens. Anal. Quantum Probab. Relat. Top. 8 (2005), 593-603.

[36] Ikeda, N. and Watanabe, S.: Stochastic Differential Equations and Diffusion Processes. NorthHolland/Kodansha, Amsterdam/Tokyo (1989).

[37] Jiřina, M.: Stochastic branching processes with continuous state space. Czechoslovak Math. J. 8 (1958), 292-313.

[38] Jiřina, M.: Branching processes with measure-valued states. In: Trans. Third Prague Conf. Information Theory, Statist. Decision Functions, Random Processes (1964), 333-357. Publ. House Czech. Acad. Sci., Prague.

[39] Kawazu, K. and Watanabe, S.: Branching processes with immigration and related limit theorems. Theory Probab. Appl. 16 (1971), 36-54.

[40] Le Gall, J.-F.: Spatial Branching Processes, Random Snakes and Partial Differential Equations. Lectures in Mathematics ETH Zürich, Birkhäuser Verlag, Basel (1999).

[41] Le Gall, J.-F. and Le Jan, Y.: Branching processes in Lévy processes: The exploration process. Ann. Probab. 26 (1998), 213-252.

[42] Li, Z.H.: Integral representations of continuous functions. Chinese Sci. Bull. (Chinese Ed.) 36 (1991), 81-84 / (English Ed.) 36 (1991), 979-983. [Preprint form: math.bnu.edu.cn/ ${ }^{\sim}$ lizh]

[43] Li, Z.H.: Measure-valued branching processes with immigration. Stochastic Process. Appl. 43 (1992), 249-264.

[44] Li, Z.H.: Convolution semigroups associated with measure-valued branching processes. Chinese Sci. Bull. (Chinese Ed.) 40 (1995), 2018-2021 / (English Ed.) 41 (1996), 276-280. [Preprint form: math. bnu.edu.cn ${ }^{\sim}$ lizh]

[45] Li, Z.H.: Immigration structures associated with Dawson-Watanabe superprocesses. Stochastic Process. Appl. 62 (1996), 73-86.

[46] Li, Z.H.: Immigration processes associated with branching particle systems. Adv. Appl. Probab. 30 (1998), 657-675.

[47] Li, Z.H.: Measure-valued immigration diffusions and generalized Ornstein-Uhlenbeck diffusions. Acta Mathematicae Applicatae Sinica 15 (1999), 310-320.

[48] Li, Z.H.: Asymptotic behavior of continuous time and state branching processes. J. Austral. Math. Soc. Ser. A 68 (2000), 68-84.

[49] Li, Z.H.: Ornstein-Uhlenbeck type processes and branching processes with immigration. J. Appl. Probab. 37 (2000), 627-634.

[50] Li, Z.H.: Skew convolution semigroups and related immigration processes. Theory Probab. Appl. 46 (2002), 274-296.

[51] Li, Z.H.: A limit theorem of discrete Galton-Watson branching processes with immigration. J. App. Probab. To appear (2005). [Preprint form: math.bnu.edu.cn $/{ }^{\sim}$ lizh] 
[52] Li, Z.H. and Shiga T.: Measure-valued branching diffusions: immigrations, excursions and limit theorems. J. Math. Kyoto Univ. 35 (1995), 233-274.

[53] Li, Z.H. and Wang, Z.K.: Measure-valued branching process and immigration processes. Adv. Math. (China) 28 (1999), 105-134.

[54] Li, Z.H. and Zhang, M.: Fluctuation limit theorems of immigration superprocesses with small branching. Statist. Probab. Letters. In press (2005).

[55] Linde, W.: Probability in Banach Spaces - Stable and Infinitely Divisible Distributions. Wiley, New York (1986).

[56] van Neerven, J.M.A.M.: Continuity and representation of Gaussian Mehler semigroups. Potential Anal. 13 (2000), 199-211.

[57] Pakes, A.G.: Some limit theorems for continuous-state branching processes. J. Austral. Math. Soc. Ser. A 44 (1988), 71-87.

[58] Pakes, A.G.: Revisiting conditional limit theorems for mortal simple branching processes. Bernoulli 5 (1999), 969-998.

[59] Pitman, J. and Yor, M.: A decomposition of Bessel bridges. Z. Wahrsch. verw. Geb. 59 (1982), 425-457.

[60] Röckner, M. and Wang, F.Y.: Harnack and functional inequalities for generalized Mehler semigroups. J. Funct. Anal. 203 (2003), 237-261.

[61] Sato, K.: Lévy Processes and Infinitely Divisible Distributions. Cambridge University Press, Cambridge (1999).

[62] Schmuland, B. and Sun W.: On the equation $\mu_{t+s}=\mu_{s} * T_{s} \mu_{t}$. Statist. Probab. Letters 52 (2001), 183-188.

[63] Schaefer, H.H.: Topological Vector Spaces. Springer-Verlag, New York (1980).

[64] Sharpe, M.J.: General Theory of Markov Processes. Academic Press, New York (1988).

[65] Shiga, T. and Watanabe, S.: Bessel diffusions as a one-parameter family of diffusion processes. $Z$. Wahrsch. verw. Geb. 27 (1973), 37-46.

[66] Walsh, J.B.: An Introduction to Stochastic Partial Differential Equations. In: Lect. Notes Math. 1180 (1986), 226-439, Springer-Verlag, New York.

[67] Wang, F.Y.: Functional Inequalities, Markov Processes, and Spectral Theory. Science Press, Beijing (2005).

[68] Wang, F.Y.: The stochastic order and critical phenomena for surperprocesses. Infin. Dimens. Anal. Quantum Probab. Relat. Top. To appear (2005).

[69] Wang, F.Y.: Dimension-free Harnack inequalities and applications. Front. Math. China 1 (2006), ??-??.

[70] Wang, H.: State classification for a class of measure-valued branching diffusions in a Brownian medium. Probab. Th. Rel. Fields 109 (1997), 39-55.

[71] Watanabe, S.: A limit theorem of branching processes and continuous state branching processes. $J$. Math. Kyoto Univ. 8 (1968), 141-167. 
[72] Zhang, M.: Large deviation for super-Brownian motion with immigration. J. Appl. Probab. 41 (2004), 187-201.

[73] Zhang, M.: Moderate deviations for super-Brownian motion with immigration. Sci. China Ser. A 47 (2004), 440-452.

[74] Zhang, M.: Moderate deviation principles for the occupation time process of a super-Brownian motion with immigration. Chinese J. Contemp. Math. 26 (2005), 61-70.

[75] Zhang, M.: On the large deviation for Brownian branching particle system. J. Appl. Probab. 42 (2005). To appear. 